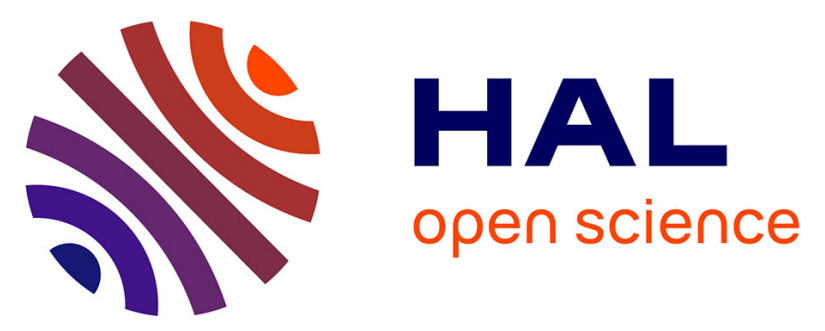

\title{
Structural characterisation of BaTiO3 thin films deposited on SrRuO3/YSZ buffered silicon substrates and silicon microcantilevers
}

Héloïse Colder, B. Domengès, Corentin Jorel, P. Marie, M. Boisserie, Samuel Guillon, Liviu Nicu, Alice Galdi, Laurence Méchin

\section{To cite this version:}

Héloïse Colder, B. Domengès, Corentin Jorel, P. Marie, M. Boisserie, et al.. Structural characterisation of BaTiO3 thin films deposited on $\mathrm{SrRuO} / \mathrm{YSZ}$ buffered silicon substrates and silicon microcantilevers. Journal of Applied Physics, 2014, 115 (5), pp.053506. 10.1063/1.4863542 . hal-00977777

\section{HAL Id: hal-00977777 https://hal.science/hal-00977777}

Submitted on 11 Apr 2014

HAL is a multi-disciplinary open access archive for the deposit and dissemination of scientific research documents, whether they are published or not. The documents may come from teaching and research institutions in France or abroad, or from public or private research centers.
L'archive ouverte pluridisciplinaire HAL, est destinée au dépôt et à la diffusion de documents scientifiques de niveau recherche, publiés ou non, émanant des établissements d'enseignement et de recherche français ou étrangers, des laboratoires publics ou privés. 


\title{
Structural characterisation of $\mathrm{BaTiO}_{3}$ thin films deposited on $\mathrm{SrRuO}_{3} / \mathrm{YSZ}$ buffered silicon substrates and silicon microcantilevers
}

\author{
H. Colder, ${ }^{1}$ B. Domengès, ${ }^{2}$ C. Jorel, ${ }^{1, a)}$ P. Marie,${ }^{3}$ M. Boisserie,,${ }^{3}$ S. Guillon, ${ }^{4}$ L. Nicu, ${ }^{4}$ \\ A. Galdi, ${ }^{1,5}$ and L. Méchin ${ }^{1}$ \\ ${ }^{1}$ GREYC, UMR 6072, CNRS, ENSICAEN, UCBN, 6 bd du Maréchal Juin, 14050 Caen Cedex, France \\ ${ }^{2}$ LAMIPS, CRISMAT-NXP Semiconductors-Presto Engineering laboratory, CNRS-UMR 6508, ENSICAEN, \\ UCBN, 2 rue de la Girafe, 14000 Caen, France \\ ${ }^{3}$ CIMAP, UMR 6252, CNRS, ENSICAEN, UCBN, CEA, 6 bd du Maréchal Juin, 14050 Caen Cedex, France \\ ${ }^{4}$ LAAS, CNRS, Univ de Toulouse, 7 avenue du Colonel Roche, 31400 Toulouse, France \\ ${ }^{5}$ Department of Industrial Engineering, CNR-SPIN Salerno, Università di Salerno, 84084 Fisciano, Salerno, \\ Italy
}

(Received 18 October 2013; accepted 16 January 2014; published online 3 February 2014)

\begin{abstract}
We report on the progress towards an all epitaxial oxide layer technology on silicon substrates for epitaxial piezoelectric microelectromechanical systems. (101)-oriented epitaxial tetragonal $\mathrm{BaTiO}_{3}$ (BTO) thin films were deposited at two different oxygen pressures, $5.10^{-2}$ mbar and $5.10^{-3} \mathrm{mbar}$, on $\mathrm{SrRuO}_{3} /$ Yttria-stabilized zirconia (YSZ) buffered silicon substrates by pulsed laser deposition. The YSZ layer full (001) orientation allowed the further growth of a fully (110)-oriented conductive $\mathrm{SrRuO}_{3}$ electrode as shown by X-ray diffraction. The tetragonal structure of the BTO films, which is a prerequisite for the piezoelectric effect, was identified by Raman spectroscopy. In the BTO film deposited at $5.10^{-2}$ mbar strain was mostly localized inside the BTO grains whereas at $5.10^{-3}$ mbar, it was localized at the grain boundaries. The BTO/SRO/YSZ layers were finally deposited on $\mathrm{Si}$ microcantilevers at an $\mathrm{O}_{2}$ pressure of $5.10^{-3}$ mbar. The strain level was low enough to evaluate the BTO Young modulus. Transmission electron microscopy (TEM) was used to investigate the epitaxial quality of the layers and their epitaxial relationship on plain silicon wafers as well as on released microcantilevers, thanks to Focused-Ion-Beam TEM lamella preparation. C 2014 AIP Publishing LLC. [http://dx.doi.org/10.1063/1.4863542]
\end{abstract}

\section{INTRODUCTION}

Piezoelectric thin films, such as $\mathrm{PbZr}_{1-\mathrm{x}} \mathrm{Ti}_{\mathrm{x}} \mathrm{O}_{3}$ (PZT), $\mathrm{SrBi}_{2} \mathrm{Ta}_{2} \mathrm{O}_{9}$, and $\mathrm{BaTiO}_{3}$ (BTO), have drawn attention due to their great potential for practical use in sensors, actuators and energy harvesting. ${ }^{1}$ When deposited in the form of a thin epitaxial film, piezoelectric films present enhanced ferroelectric properties. ${ }^{2-4}$ Achieving high piezoelectric coefficients is needed in order to realize high sensitivity sensors and large displacement. It is indeed well established that the piezoelectricity is appealing for electromechanical transduction at the microscale as it allows energy conversion from the mechanical to the electrical domain and vice-versa. Before exploiting epitaxial piezoelectrical films in operational piezoelectric Micro-ElectroMechanical Systems (MEMS) or Nano-ElectroMechanical Systems (NEMS), a careful optimization has to be done since the epitaxial growth may also induce additional unwanted mechanical stresses. ${ }^{5,6}$ These stresses may damage the MEMS structure and performance. ${ }^{7,8}$

Epitaxial MEMS incorporating PZT or $\mathrm{Pb}\left(\mathrm{Mg}_{1 / 3} \mathrm{Nb}_{2 / 3}\right) \mathrm{O}_{3}$ $-\mathrm{PbTiO}_{3}$ (PMN-PT) have been recently reported in literature. ${ }^{9-11}$ BTO also has promising PE properties, such as a bulk piezoelectric coefficient $d_{33}=190$ pC.N ${ }^{-1}$ (Ref. 12) and a dielectric coefficient $\varepsilon_{33}=1700$ (Ref. 13) at room temperature and it is

\footnotetext{
a) Author to whom correspondence should be addressed. Electronic mail: corentin.jorel@unicaen.fr
}

particularly attractive because of its lead free and environmentally friendly characteristics. BTO was therefore used as the piezoelectric material in the present work. Metal films such as $\mathrm{Pt}$ or $\mathrm{Pt} / \mathrm{Ti}$ are commonly used due to their excellent conducting properties and because they allow the growth of subsequent functional oxides. Nevertheless, when BTO films are directly deposited on metal electrodes, a severe reduction of their dielectric constant, serious fatigue degradation and film cracking are observed ${ }^{14,15}$ mostly due to the existence of an interfacial "dead layer." 16 To replace the metal electrode, conductive oxides have been investigated, ${ }^{17-20}$ resulting in a better aging of the devices mainly thanks to their capacity to absorb oxygen vacancies at the interfaces between the electrode and the ferroelectric layer. ${ }^{19}$ Among them, we chose $\mathrm{SrRuO}_{3}$ (SRO) oxide ${ }^{9-11,20}$ because of its similar structure and reasonably good lattice matching with $\mathrm{BTO}^{21}$ thus enabling its further epitaxial growth. Last, Silicon (Si) or Silicon-On-Insulator (SOI) substrates have to be used in order to use the silicon micromachining techniques for releasing the structures (bridges, cantilevers, membranes, etc). However, it is difficult to prepare epitaxial oxide films by direct deposition on Si substrates due to the large lattice mismatch, the severe diffusion between the deposited oxide films and $\mathrm{Si}$, and the amorphous native silicon oxide. Furthermore, as $\mathrm{Si}$ and SRO show a high chemical reactivity, the SRO films directly deposited on $\mathrm{Si}$ are often polycrystalline. $^{22}$ To solve these problems, a buffer layer is required. Among the different materials that have been used 
as buffer layer for the epitaxial growth of BTO, one can commonly find $\mathrm{SrTiO}_{3}$ and Yttria Stabilized Zirconia (YSZ). ${ }^{23-26}$

In the present study, Yttria Stabilized Zirconia (YSZ) buffer layers were used to grow epitaxial BTO films on Si (001) substrates using SRO conducting electrodes. Experimental details are given in section II. The crystalline properties of the YSZ buffer layers and of the SRO/YSZ layers were investigated by X-Ray diffraction (XRD), Atomic Force Microscopy (AFM), spectroscopic ellipsometry, and X-ray reflectivity (XRR) in Sec. III. Raman spectroscopy and XRD were used to determine the structural properties of the BTO layers deposited on SRO/YSZ on bare $\mathrm{Si}$ (001) substrates in Sec. IV. The influence of oxygen pressure on the growth direction and on the strain repartition is particularly discussed. Section V presents the Transmission and Scanning Transmission Electron Microscopy (TEM-STEM) study of the layer stacking and includes the out-of-plane and in-plane epitaxial relationships. The BTO/SRO/YSZ multilayers were deposited on suspended microcantilevers. BTO Young modulus was evaluated and the material quality was checked by Scanning Electron Microscopy (SEM), Fabry-Perot interferometry and TEM (Sec. VI). Conclusions and perspectives are finally given in Sec. VII.

\section{EXPERIMENTAL DETAILS}

The BTO, SRO, and YSZ layers were deposited in a single run by PLD using a $\mathrm{KrF}$ excimer laser $(248 \mathrm{~nm})$. After the introduction of the $\mathrm{N}$-doped $\mathrm{Si}(001)$ substrates in the deposition chamber, the latter was pumped down to $10^{-5}$ mbar and the substrate temperature was increased to $730^{\circ} \mathrm{C}$. The YSZ buffer layers were deposited at a temperature of $730{ }^{\circ} \mathrm{C}$ in $\mathrm{O}_{2}$ pressure of $10^{-4} \mathrm{mbar}$, with a laser pulse rate of $3 \mathrm{~Hz}$ and an energy of $220 \mathrm{~mJ}$, similarly to our previous works using epitaxial YSZ layers on $\mathrm{Si}^{27-30}$ PLD experimental details concerning the subsequent layers are given in Table I. After deposition, the oxygen pressure was raised to 700 mbar and the substrate temperature was decreased at the rate of $10^{\circ} \mathrm{C} / \mathrm{min}$ down to room temperature.

Arrays of silicon microcantilevers were fabricated using SOI substrates and a UV stepper photo repeater. The use of SOI wafers with $340 \mathrm{~nm}$ thick p-type $\mathrm{Si}\left(10^{15} \mathrm{at}^{\mathrm{cm}} \mathrm{cm}^{-3}\right)$ on a $1 \mu \mathrm{m}$-thick buried $\mathrm{SiO}_{2}$ oxide (BOX) layer over $525 \mu \mathrm{m}$ thick $\mathrm{Si}$ substrate (from Soitec, France) ensured the production of cantilevers with a controllable thickness (since fabricated in the top silicon layer) and the release of the cantilevers in aqueous solution without structure collapsing and sticking issues (by using the BOX layer as a sacrificial one). A UV stepper photo repeater (I Line CANON FPA 3000 i4/i5, N.A. 0.63 ) was used to pattern the shape of the microcantilevers via a $600 \mathrm{~nm}$ thick positive photoresist layer (ECI). After developing the photoresist, the top silicon layer was vertically etched by reactive ion etching (RIE, Alcatel AMS4200) until the intermediate $\mathrm{SiO}_{2}$ layer appeared. The sacrificial $\mathrm{SiO}_{2}$ was then etched by dipping the entire wafer in a buffered hydrofluoric acid (BHF) solution in order to release the microcantilevers.

Several techniques were used to analyze the crystalline properties of the layers. The crystallographic structure of the thin films was characterized by XRD $\theta-2 \theta$ scans (XRD Philips XPERT HPD Pro Device) using $\mathrm{CuK}_{\alpha}$ radiation (wavelength 1.5419 Á). Since XRD does not enable to conclude definitively on the cubic or tetragonal crystalline structure of BTO, Raman spectroscopy was also used. Raman scattering measurements were carried out at room temperature in backscattering geometry with a Jobin Yvon LabRam Raman Confocal microscope. A $588 \mathrm{~nm}$ Ar ion laser with a power of about $6 \mathrm{~mW}$ and an objective of $\times 100$ were used for the Raman investigations. The thickness of the layers was investigated by means of X-ray reflectometry and phase-modulated spectroscopic ellipsometry. The ellipsometry data were collected using of a Jobin-Yvon ellipsometer (UVISEL) where the incident light was scanned in the range of $1.5-4.5 \mathrm{eV}$ under an incident angle of $66.3^{\circ}$. The fitting of the experimental data was performed using DeltaPsi2 software. ${ }^{31}$ AFM microscopy was performed in tapping mode using a Nanoscope III Multimode from Digital Instruments.

TEM-STEM observations of the complete layer stack were performed in a JEM 2010 TEM-STEM-FEG, operating at $200 \mathrm{kV}$, equipped with a high-resolution objective lens $\left(\mathrm{C}_{\mathrm{s}}=1 \mathrm{~mm}\right)$, JEOL High-Angle-Aperture-Dark-Field detector (HAADF), Energy Dispersive X-Ray Spectroscopy (EDXS) PHOENIX system, and tilting-rotating sample holder allowing the orientation of layer interfaces towards the electron beam. Transmission Electron Microscopy (TEM) lamellae were prepared either in a JEOL Ion Slicer using $\mathrm{Ar}^{+}$ion beam milling or in a Focused Ion Beam (FIB) system when precise location of the thin lamella was necessary (bridge). FIB milling was performed in a single beam FEI 200XP system $\left(30 \mathrm{kV} \mathrm{Ga}^{+}\right.$ion beam and Pt deposition chemistry) adapted to lift-out in situ preparation technique. ${ }^{32,33}$ In that case, in order to protect the bridge top layer from FIB irradiation damage, a thin layer of Au-Pd (about $50 \mathrm{~nm}$ ) was deposited in an evaporator. TEM is a very local characterization technique and the final lamella size ( $\sim 20 \mu \mathrm{m}$ in length) is much smaller than the sample one $\left(1 \times 1 \mathrm{~cm}^{2}\right)$. Those appeared to exhibit slight differences in colour (from greenish-yellow to yellowish-green) under optical microscopy observations, and several lamellae were then prepared. After TEM characterization, it could be interpreted to be linked to variations of the BTO layer thickness. This phenomenon is not rare with PLD deposition technique. Nevertheless,

TABLE I. Deposition conditions of all layers using PLD.

\begin{tabular}{lccccc}
\hline \hline Layer & Expected thickness $(\mathrm{nm})$ & Substrate temperature $\left({ }^{\circ} \mathrm{C}\right)$ & $\mathrm{O}_{2}$ pressure $(\mathrm{mbar})$ & Laser pulse rate $(\mathrm{Hz})$ & Laser energy $(\mathrm{mJ})$ \\
\hline YSZ & 70 & 730 & $10^{-4}$ & 3 & 220 \\
SRO & 20 & 730 & 0.2 & 2 & 170 \\
BTO & $50-150$ & 730 & $5.10^{-3}$ or $5.10^{-2}$ & 3 & 200 \\
\hline \hline
\end{tabular}


following TEM study results (microstructures, relative orientations...) apply whatever the BTO layer thickness is.

The BTO/SRO/YSZ multilayers were deposited onto freestanding silicon-based microcantilevers. Accordingly with the dynamic interferometry technique developed by Carr and Craighead for measuring the Young modulus, ${ }^{34}$ devices were mounted on a small piezoelectric disk, electrically actuated by a network analyzer (Agilent 4395A) and coupled to an in-house high-frequency amplifier, so that the entire substrate was vibrating. The devices were placed inside a vacuum chamber pumped down to $10^{-6} \mathrm{mbar}$ at room temperature. A $30 \mathrm{~mW}$ He-Ne laser (Melles Griot) was focused on the microstructures using a beam expander and a long working distance microscope objective (20X, N.A. 0.28 ), leading to a $3 \mu \mathrm{m}$ minimal beam size on the cantilever. Interferences were detected by a photodetector (New Focus 1601) connected to the network analyzer to track the cantilever response at the excitation frequency. The vibrations of the microcantilevers resulted in a periodic change of the interference intensity, which corresponds to the resonant frequency of the microcantilevers.

\section{STRUCTURAL AND ELECTRICAL PROPERTIES OF SRO ELECTRODES ON YSZ/SI (001) SUBSTRATES}

The YSZ buffer layer plays a crucial role in the epitaxial growth of the whole BTO/SRO/YSZ multilayer on $\mathrm{Si}$. $\mathrm{Zr}$ is expected to react with $\mathrm{SiO}_{2}$ the amorphous native oxide existing at the silicon surface as follows: $\mathrm{Zr}+2 \mathrm{SiO}_{2} \rightarrow \mathrm{ZrO}_{2}+2$ $\mathrm{SiO}$ where $\mathrm{SiO}$ is volatile, thus allowing an epitaxial growth on the crystalline Si surface. The (001)-oriented Si substrate should impose a cube-on-cube growth of (001) YSZ layers. ${ }^{27}$ Indeed, YSZ buffer layer is (001)-oriented as revealed by XRD scans in the $\theta-2 \theta$ configuration in Figure 1.

To measure the thickness of the YSZ layer, spectroscopic ellipsometry was used because it does not require a special environment and it is a fast, sensitive and non-destructive method for film characterization. Data were fitted using a dispersion law, which is based on the Forouhi-Bloomer model elaborated for amorphous semiconductors and insulating

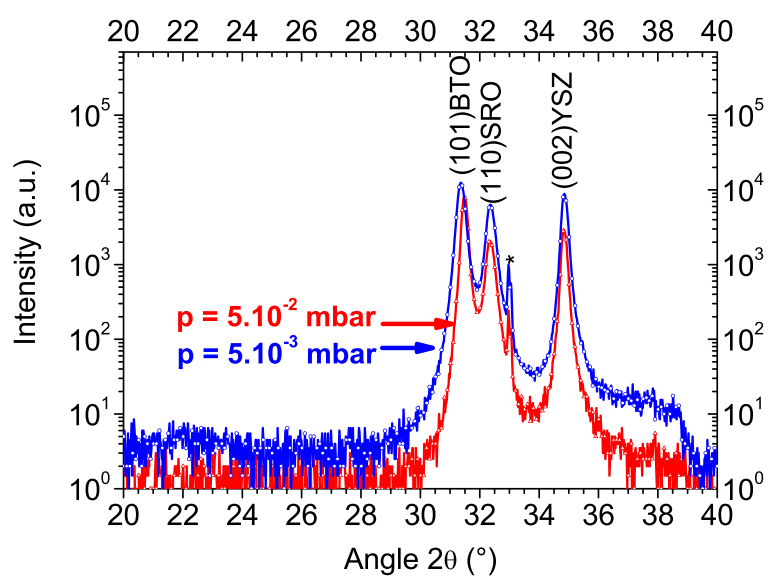

FIG. 1. X-Ray diffractograms in the $\theta-2 \theta$ configuration of BTO thin films deposited at $p=5.10^{-2}$ mbar (red) and $\mathrm{p}=5.10^{-3}$ mbar (blue) on buffered $\mathrm{Si}$ substrate. The peaks due to the buffer layers are identified as SRO (110) and YSZ (002) (the peak marked $*$ is the $\mathrm{Si}(004)$ peak at $\lambda / 2$ ). materials ${ }^{35}$ using an improved parameterization. ${ }^{36}$ The model structure of the film was optimized by least square refinement approach $\left(\chi^{2}\right)$ from a fitting of the experimental data. For a good correspondence between the fitting curve and the experimental data an interfacial $\mathrm{SiO}_{2}$ layer between the YSZ layer and Si substrate is necessary. ${ }^{37}$ As a result, the thickness of each layer is estimated to be $70.3 \pm 7 \mathrm{~nm}$ for YSZ and $2.9 \pm 0.3 \mathrm{~nm}$ for $\mathrm{SiO}_{2}$. XRR measurements were also performed on the same YSZ/Si layers and fitted with Simulreflec software. ${ }^{38}$ The value of $69.5 \mathrm{~nm}$ for YSZ was found, which is in good agreement with the ellipsometry analysis. The root mean square (RMS) roughness of the YSZ layer deposited on Si was measured to be $0.3 \mathrm{~nm}$ in $10 \mu \mathrm{m} \times 10 \mu \mathrm{m}$ AFM images, which confirms the $0.32 \mathrm{~nm}$ value obtained from XRR data.

The structural and morphological properties of the YSZ layer presented above allow the subsequent growth of SRO and BTO. We chose SRO as the conductive epitaxial bottom electrode layer. Having an epitaxial bottom electrode is essential in order to induce the further epitaxial growth of the piezoelectric layer indeed. In agreement with previous work, ${ }^{2,30}$ pseudo cubic (110)-oriented SRO layers were obtained on top of (001) YSZ/Si (Figure 1). Dekkers et al. ${ }^{2}$ assumed that the $45^{\circ}$ rotated cube on cube SRO arrangement is preferred because of the specific stoichiometric ratio of the elements during the growth. As $\mathrm{Sr}$ and $\mathrm{Ru}$ are present early in the growth, the first layers contain both elements in equal amounts and consequently the (110) growth orientation is promoted. The $c$ parameter of the pseudo-cubic SRO in multilayers is estimated from the X-Ray peak position. It is evaluated to be $3.914 \AA$, close to that of the bulk cubic SRO, $3.910 \AA$ A. The SRO layers present a good crystallinity and a low level of strain. Moreover, no additional XRD peak is detected. In spite of the well-known strong chemical interaction $^{39}$ between SRO and Si, there is no evidence of any reaction between the two materials, thus validating the chemical barrier efficiency of the YSZ layer.

The SRO thicknesses were deduced from spectroscopic ellipsometry measurements by introducing an additional layer as done in the previous model used for YSZ layers on Si. The best fit correspondence is obtained for the thicknesses of $23 \pm 2$, $60 \pm 6$, and $2 \pm 0.2 \mathrm{~nm}$, for SRO, YSZ, and $\mathrm{SiO}_{2}$, respectively. The RMS roughness of the SRO layer is about $15 \mathrm{~nm}$.

The electrical behavior of SRO layers was investigated by measuring the resistivity as a function of temperature in a four point configuration. SRO shows a metallic-like behavior and the resistivity at $300 \mathrm{~K}$ is approximatively $1 \times 10^{-5} \Omega \cdot \mathrm{m}$ and the transition temperature (as seen by the change of slope of the resistivity versus temperature curve) is close to $160 \mathrm{~K}$ (see Ref. 30 for a more detailed study of the SRO resistivity versus temperature characteristics). These rather high values compared to bulk SRO suggest a relaxed and granular microstructure of the SRO layer, ${ }^{40,41}$ which will be confirmed by TEM observations (see below).

\section{BTO THIN FILMS ON SRO/YSZ/SI (001) BARE SUBSTRATES}

Particular care has to be taken in order to grow BTO with the good crystallographic orientation for the desired 
piezoelectric MEMS applications. At room temperature, the piezoelectric effect in BTO is due to the displacement of $\mathrm{Ti}$ along the c-axis in the BTO tetragonal structure. However, some deposition conditions can induce a pseudo-cubic crystallization of the BTO films. ${ }^{42,43}$ Because of its perfect symmetry, this cubic structure does not show any PE effect. In order to investigate the structural properties of our films, we used a combination of XRD and Raman spectroscopy. XRD does not always allow distinguishing between tetragonal and cubic oriented structures, contrary to Raman spectroscopy. ${ }^{44}$ When the difference between $c$ and $a$ parameters is important, some XRD peaks are split: for example (110) split in (110) and (101). If there is a preferential orientation (for example (110)), only a single peak is generated. The problem is that this peak can also be associated to a cubic structure. On the contrary the cubic structure is undetectable with Raman spectroscopy ${ }^{45}$ whereas the tetragonal structure induces some peaks. So Raman spectroscopy allows to be sure of the presence of the BTO tetragonal structure.

Figure 2 shows the Raman spectra of the BTO films deposited at two different oxygen pressures $\left(5.10^{-2}\right.$ and $5.10^{-3}$ mbar) on SRO/YSZ/Si. With the reference spectrum of the $\mathrm{SRO} / \mathrm{YSZ} / \mathrm{Si}$ stack, the vibrations due to BTO films can easily be identified. On both $\mathrm{BTO} / \mathrm{SRO} / \mathrm{YSZ} / \mathrm{Si}$ spectra, one small peak at $185 \mathrm{~cm}^{-1}\left(\mathrm{~A}_{1}\left(\mathrm{TO}_{1}\right)\right)$ and two well-defined peaks, at $305 \mathrm{~cm}^{-1}\left(\mathrm{E}\left(\mathrm{TO}_{2}\right)\right)$ and around $700-730 \mathrm{~cm}^{-1}$ $\left(\mathrm{A}_{1}\left(\mathrm{LO}_{3}\right)\right)$ are observed and are associated to tetragonal BTO. ${ }^{43,46}$ Based on the work of Frey and Payne, ${ }^{47}$ the peak at $185 \mathrm{~cm}^{-1}$ is assigned to the BTO tetragonal structure. The BTO film deposited at $5.10^{-3}$ mbar is characterized by a peak around $730 \mathrm{~cm}^{-1}$, i.e., just near the phonon frequencies of the BTO single crystal $\left(728 \mathrm{~cm}^{-1}\right),{ }^{48}$ whereas, at $5.10^{-2} \mathrm{mbar}$, the peak clearly shifts to $700 \mathrm{~cm}^{-1}$. It is known that the phonon frequencies shift towards higher/lower values depending upon the tensile/compressive nature of the out-of-plane strain. ${ }^{49}$ This means that the strain is reduced when the BTO film is deposited at $5 \cdot 10^{-3}$ mbar. At higher pressure, the BTO film undergoes an out-of-plane compressive strain. Otherwise BTO films are well-known to have a high oxygen vacancy density. Guo et al. ${ }^{50}$ investigated BTO thin films which were poor in oxygen (rich in oxygen

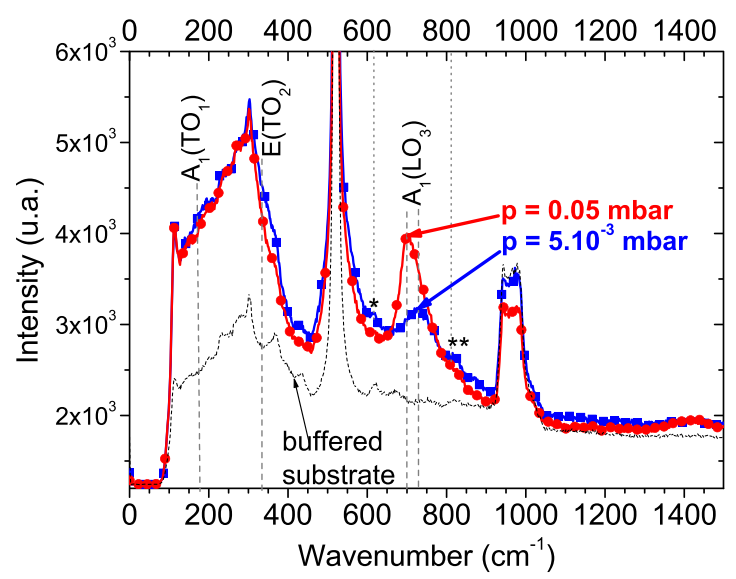

FIG. 2. Raman spectra of a buffered Si substrate and of BTO thin films deposited at $p=5.10^{-2}$ mbar (red) and at $p=5.10^{-3}$ mbar (blue) on buffered Si substrate. The strain induced peaks are identified by * and **. vacancies). They observed two broad and weak peaks at 943 and $1039 \mathrm{~cm}^{-1}$ only for films poor in oxygen. They associated these peaks to the BTO lattice distortion due to oxygen deficiency. In Figure 2, these peaks are not detected, which allows us to conclude that the tetragonal structure of BTO is not distorted due to oxygen vacancies. This parameter is very important since it should favour a good electrical response to mechanical excitation (in functioning MEMS).

Two additional peaks, marked by asterisks, are also observed. First, in both spectra, the peaks around $820 \mathrm{~cm}^{-1}$ (marked by $* *$ in Figure 2) might originate from the strain at the SRO/BTO interface due to the lattice parameter mismatch, as Guo et al. noticed concerning the $\mathrm{BTO} / \mathrm{MgO}$ interface. ${ }^{50}$ Second, the peak at $618 \mathrm{~cm}^{-1}$ (marked by $*$ in Figure 2) is only detected for BTO films grown at $5.10^{-3}$ mbar of $\mathrm{O}_{2}$. Naik et al. also observed a peak at $620 \mathrm{~cm}^{-1}$ on Raman spectra of their BTO thin films. ${ }^{51}$ They attributed it to a strain localized in the grain-boundary regions. This interpretation is also supported by their previous work on BTO nanocrystals. ${ }^{52}$

From the comparison with the peak positions and their interpretation in the literature, our Raman spectroscopy measurements allowed us to draw some conclusions. First, both BTO films have a pure tetragonal structure without a strain signature due to oxygen vacancies and they contain a tensile strain at the BTO/SRO interface. Second, the (out-ofplane) strain repartition in the BTO film changes with the oxygen pressure. Indeed in the film deposited at $5.10^{-2}$ mbar, compressive strain is concentrated in the BTO crystal structure (the strain is localized inside the grains ${ }^{48}$ ). On the contrary, in the BTO film deposited at $5.10^{-3}$ mbar, the strain is most probably located at the grain boundaries. ${ }^{50}$

Considering the XRD $\theta-2 \theta$ scans of Figure 1, BTO films seem to grow on the (110)-oriented SRO with preferential orientations (110) or (101). By increasing the pressure from $5.10^{-3}$ mbar to $5.10^{-2}$ mbar, the BTO peak shifts from $31.37^{\circ}$ to $31.49^{\circ}$. On the one hand, the value of this shift, $0.12^{\circ}$, is not far from $0.18^{\circ}$, the difference between the (101) and (110) peak positions in BTO single tetragonal crystal. ${ }^{53}$ If we attribute the $31.49^{\circ}$ peak to the (110) orientation of BTO phase, it would lead to an interreticular distance $d_{110}$ of $2.841 \AA$. This value is higher than that expected in BTO tetragonal structure $\left(d_{110}=2.824 \AA\right)$. It is inconsistent with the expected compressive strain which has been confirmed in Raman spectroscopy $\left(\mathrm{A}_{1}\left(\mathrm{LO}_{3}\right)\right.$ peak Figure 2$)$. On the other hand, if the $31.49^{\circ}$ peak of the $5.10^{-2}$ mbar BTO film is indexed as a (101) reflection, it corresponds to a decrease in the cell volume of about $4 \%$ due to the compressive strain localized in the grains as deduced from Raman study. Thus, it appears most probable that both BTO films are (101) oriented.

It is well-known that the preferred orientation of thin films is affected by many parameters. The substrate and buffer nature and orientation ${ }^{16,54}$ but also the surface free energies of two dimensional planes play an important role in the first stage of the growth. Since the (111) BTO plane shows the highest occupation density plane, the film should be (111)-oriented but this is seldom observed. Indeed, surface free energy also depends on the electrostatic charge. 
BTO being a highly ionic crystal, its bonds have a predominantly ionic character: $82 \%$ for the Ba-O bond and $63 \%$ for the Ti-O bond. As shown by Kim et al. ${ }^{54,55}$ the (100) equivalent planes are the unique electrical neutral planes, which explains why without oxygen during deposition ( $p=10^{-5}$ mbar) the BTO film is (001) oriented..$^{55}$ As soon as some oxygen is introduced during deposition, the preferred orientation switches to (110). ${ }^{56,57}$ In the presence of oxygen, ionic species ejected from the target surface can react and lose some energy, which implies that the film grows in the direction of the thermodynamically stable plane (110). In our deposition conditions (oxygen pressure and (110) oriented SRO below BTO), it is therefore not surprising not to have (001) but (101) oriented BTO films. As Raman spectroscopy revealed, the oxygen pressure will mainly influence the repartition of the film's strain. The BTO film deposited at $5.10^{-2}$ mbar shows compressive strain located in the BTO grains, whereas the strain in the BTO film deposited at $5.10^{-3}$ mbar is localized at the grain-boundaries. In fact, the deposition at lower pressure allows the crystal stress relaxation during the growth.

\section{TEM STUDY OF BTO/SRO/YSZ MUTLILAYERS ON SI (001)}

Figure 3 shows the main results of the TEM study performed on the complete layer stacking. These results apply to both oxygen partial pressures used for deposition of BTO layer. The YSZ layer appears well-crystallized, under the form of grains most often as large as thick (Figure 3(a)). Indeed, strain contrast and some rare Moiré patterns can be observed on bright field images. Nevertheless, the Selected Area Electron Diffraction (SAED) pattern exhibits a single common orientation of YSZ grains, which are perfectly aligned with the $\mathrm{Si}$ substrate ((Figure 3(b)), in agreement with cube on cube growth. ${ }^{27}$ This orientation relationship is observed on both $\mathrm{Si}$ and SOI substrates. Indeed, the $340 \mathrm{~nm}$ p-type SOI Si layer (SOI) is slightly disorientated $\left(\sim 2\right.$ to $\left.3^{\circ}\right)$ with respect to $\mathrm{Si}$ substrate and in that case, YSZ [001] is parallel to the SOI silicon layer
[001] axis and not to Si substrate one. This epitaxy confirms that the thin $\mathrm{SiO}_{2}$ layer (4-5 nm), which can be seen (small arrow on (Figure 3(a)), has grown after the YSZ epitaxy. Nevertheless, a tiny disorientation of the grains is deduced from the slight splitting of the diffraction spots (pointed on Figure 3(b)). The layer thickness can be measured locally on different points of the TEM lamellae (several microns wide). The sample presented in Figure 3 is deposited on bulk Si and has a thicker $100 \mathrm{~nm}$ YSZ film. Nevertheless, the thickness of the interfacial silicon oxide layer stays close to $5 \mathrm{~nm}$ and does not show any dependence on the YSZ thickness. The lamellae prepared on a SOI substrate show the expected $\sim 70 \mathrm{~nm}$ thick YSZ film (same as the one controlled by ellipsometry) and observations of one prepared on a patterned area are given in Sec. VI.

Though SRO and BTO layers appear well-crystallized and aligned with respect to each other and to the lower layers on SAED pattern (Figure 3(c)), they can hardly be distinguished on bright field images. Selected Area Electron diffraction is neither precise enough to discriminate between pseudoand true symmetry nor to find the actual BTO film orientations in a tetragonal cell. Thus, the diffraction dots indexation is proposed based on the complementary previous results (see Secs. III and IV), that is pseudo-cubic structure of the SRO layer and (101)-orientation of the tetragonal BTO one. The following orientation relationship between subsequent layers can be given (in the reciprocal space) parallelly to growth direction: $\quad[001]{ }^{*}{ }_{\mathrm{Si}} / /[001]{ }^{*}{ }_{\mathrm{Ys}} / /[110]{ }_{\mathrm{SRO}} / /[101]{ }_{\mathrm{BTO}}, \quad$ and in-plane: $[110]{ }_{\mathrm{Si}} / /[110]{ }^{*}{ }_{\mathrm{YSZ}} / /[1 \overline{1} 2]{ }^{*} \mathrm{SRO} / /[12 \overline{1}]{ }^{*}{ }_{\mathrm{BTO}}$.

Besides, HAADF STEM image and EDXS line profile across the complete layer stacking show the interpenetration of SRO and BTO layers in the thickness of the TEM lamella (Figure 4). This can be easily understood knowing the higher roughness of the SRO layer (AFM results) and the small size of SRO grains. It is thus difficult to measure the SRO layer thickness as it varies from 30 to $70 \mathrm{~nm}$, depending on the grain size. The important point is that it completely covers the YSZ one and acts as an electrode, as shown by its electrical characteristic. In the same way, the BTO thickness varies mostly from 110 to $155 \mathrm{~nm}$.
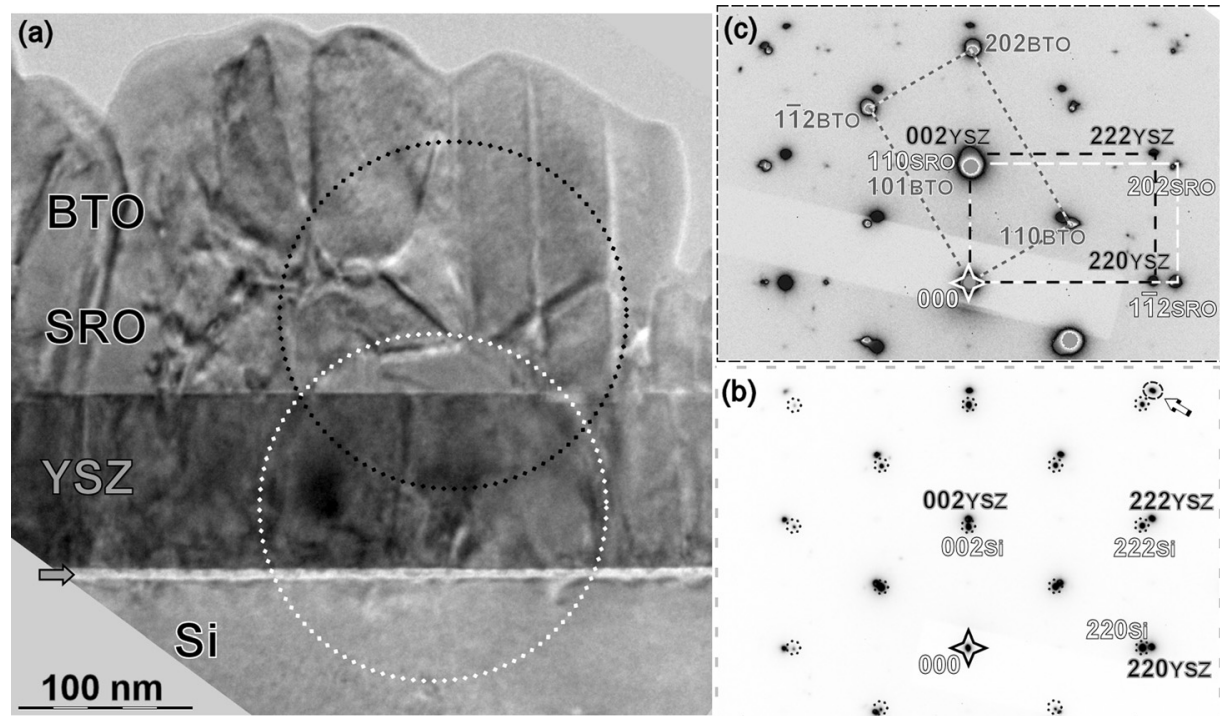

FIG. 3. (a) Medium resolution TEM image of the complete layer stacking on Si substrate, dotted circles indicate the selected areas for electron diffraction (SAED) patterns of (b) YSZ/Si, $\mathrm{Si}$ framework spots are circled and (c) $\mathrm{BTO}(0.05) / \mathrm{SRO} / \mathrm{YSZ}$, each framework is materialized through dotted rectangles, black (resp. white and grey) for YSZ (resp. SRO and BTO). 

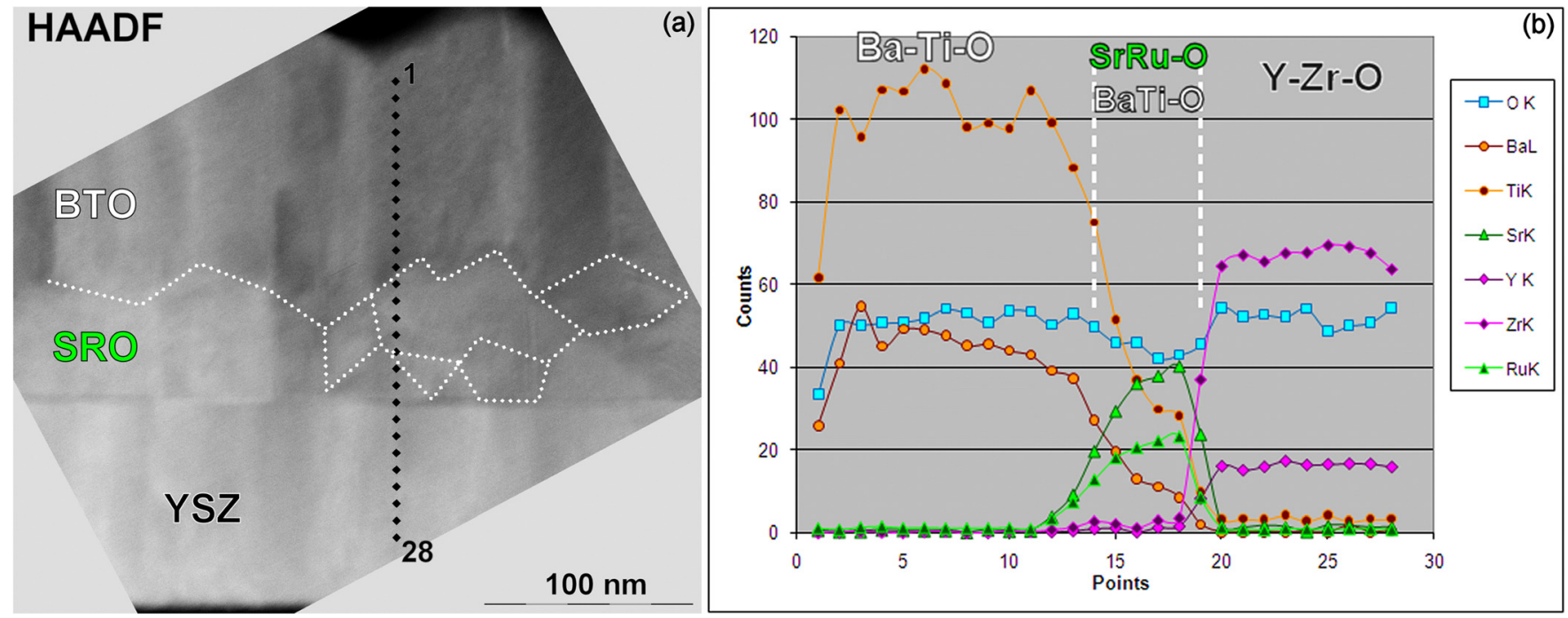

FIG. 4. (a) HAADF STEM image and (b) EDXS line profiles showing the interpenetration of SRO and BTO (deposited at 0.05 mbar $\mathrm{O}_{2}$ ) layers.
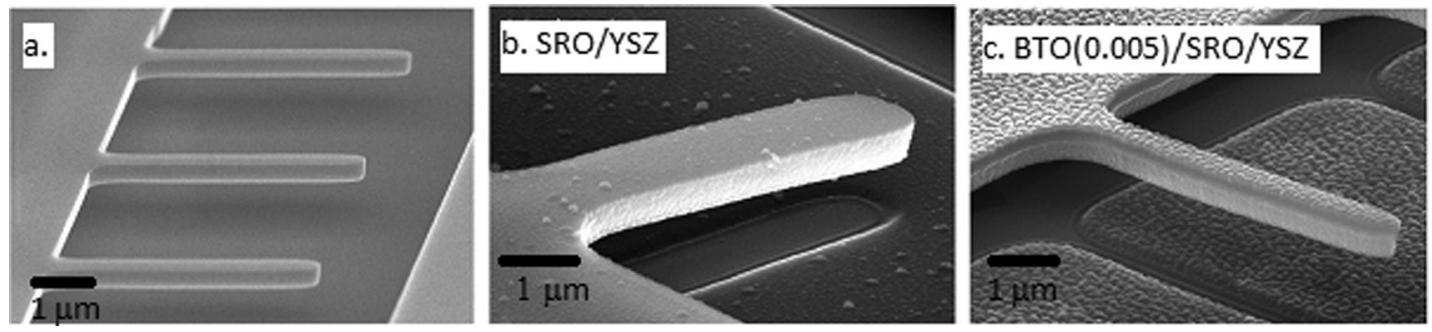

FIG. 5. SEM images of the microcantilevers (a) without any layer, (b) with a SRO/YSZ bi-layer, and (c) with a BTO/SRO/YSZ tri-layer (BTO being deposited at $5.10^{-3}$ of $\mathrm{O}_{2}$ ).

\section{BTO THIN FILMS ON SRO/YSZ ON RELEASED SILICON CANTILEVER}

The microcantilevers were processed on SOI wafers as described in Sec. II. Examples of released silicon microcantilevers are shown in Figure 5(a). The SOI wafers containing the microcantilevers were diced in $1 \mathrm{~cm}^{2}$ individual chips in order to fit the sample holder of the PLD set-up. A SRO/YSZ bi-layer was first deposited on one chip bearing the microcantilevers (Figure 5(b)) and a BTO/SRO/YSZ

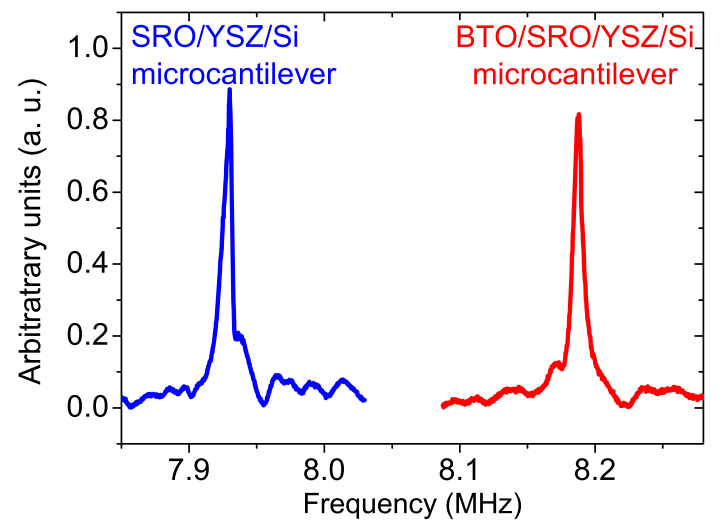

FIG. 6. Resonant frequency peaks measured on SRO/YSZ (blue, left) and on BTO/SRO/YSZ microcantilevers (red, right) corresponding to sample C Table III. tri-layer was deposited on another chip (Figure 5(c)). The $\mathrm{O}_{2}$ pressure of $5.10^{-3}$ mbar was chosen for BTO because of the absence of compressive strain within the BTO grains, which would be fatal for the piezoelectric effect. As shown in Figures 5(b) and 5(c), the microcantilevers present no crack, reduced strain and a uniform deposition of the multilayer.

The resonant frequencies corresponding to the fundamental resonant mode of vibration have been measured on both SRO/YSZ and BTO/SRO/YSZ microcantilevers (Figure 6). It can be seen that the presence of the BTO layer results in a resonant frequency shift $\Delta f$. From this shift and the equivalent stiffness of the microcantilevers ${ }^{58}$ estimated by considering Young moduli and densities of silicon, YSZ and SRO as known from literature (Table II), the BTO Young modulus $E$ value can be evaluated.

Measurements on three cantilevers (A, B, C) with different dimensions give different $E$ values, as shown in Table III. A

TABLE II. Material data used for the estimation of the BTO Young modulus.

\begin{tabular}{lcc}
\hline \hline Material & Young modulus & Density \\
\hline $\mathrm{Si}$ & $169 \mathrm{GPa}$ (Ref. 59) & $2330 \mathrm{~kg} / \mathrm{m}^{3}$ (Ref. 60) \\
YSZ & $205 \mathrm{GPa}$ (Ref. 61) & $5960 \mathrm{~kg} / \mathrm{m}^{3}$ (Ref. 62) \\
SRO & $161 \mathrm{GPa}$ (Ref. 63) & $6490 \mathrm{~kg} / \mathrm{m}^{3}$ (Ref. 63) \\
BTO & $E$ (to be calculated) & $6010 \mathrm{~kg} / \mathrm{m}^{3}$ (Ref. 53) \\
\hline
\end{tabular}


TABLE III. Data used for the estimation of the BTO Young modulus $E$ : the dimension of silicon microcantilevers without deposition (width $w$, length $l$ and thickness $t$ ), the overetching $(O E)$, the vibration frequency with the SRO/YSZ bi-layer only $\left(f_{i}\right)$ and with the BTO/SRO/YSZ tri-layer $\left(f_{f}\right)$, and the measured frequency shift $(\Delta f)$.

\begin{tabular}{lcccccccc}
\hline \hline Microcantilever & $w(\mu \mathrm{m})$ & $l(\mu \mathrm{m})$ & $t(\mathrm{~nm})$ & $O E(\mu \mathrm{m})$ & $f_{i}(\mathrm{MHz})$ & $f_{f}(\mathrm{MHz})$ & $\Delta f(\mathrm{Mhz})$ & $E(\mathrm{GPa})$ \\
\hline A & 1.1 & 10 & 300 & 1.3 & 4.31 & 4.50 & 0.19 \\
B & 1.3 & 10 & 300 & 1.3 & 4.27 & 4.34 & 260 \\
C & 1.85 & 7 & 300 & 1.3 & 7.93 & 8.19 & 0.07 & 223 \\
\hline \hline
\end{tabular}

mean BTO thickness of $110 \mathrm{~nm}$ was used for the calculations. We obtained a Young modulus value of $240 \pm 18 \mathrm{GPa}$. This average value is much higher than the value for bulk BTO $(67 \mathrm{GPa}){ }^{64,65}$ But calculated and measured $E$ values in the range of $90-147 \mathrm{GPa}$ are reported in literature for tetragonal BTO. ${ }^{66-68}$ Our measured $E$ value is still higher than literature data. This can be explained by internal strain, ${ }^{69}$ crystalline parameters ${ }^{70}$ and grain thickness. By decreasing grains size from 0.7 to $0.3 \mu \mathrm{m}$ in ceramic $\mathrm{BaTiO}_{3}, E$ increases from 168 to $186 \mathrm{GPa}$ according to Arlt et $\mathrm{al}^{71}$ Here, the grain size is smaller, which could explain our higher $E$ value. It should be noted that the in grain-boundary strain and the preferential orientation also play an important role.

TEM observations were performed on a lamella prepared along a released BTO/SRO/YSZ/Si bridge. Figure 7 shows the medium resolution image as well as the corresponding SAED pattern. In addition to the $\mathrm{BTO} / \mathrm{SRO} / \mathrm{YSZ} / \mathrm{Si}$ stack, one can observe the expected thin $\mathrm{SiO}_{2}$ layer (12 nm thick) above $\mathrm{Si}$, as also observed in our XRR and reflectometry measurements performed on BTO/SRO/YSZ on bare Si (Sec. III). In addition, an extra amorphous layer ( $38 \mathrm{~nm}$ thick) is seen in Figure 7(a). This layer does not appear homogeneous, exhibiting an amorphous-type contrast speckled with darker areas. The EDSX analyses presented in Figure 8 indicated that its composition is mainly $\mathrm{SiO}_{\mathrm{x}}$. They also revealed that this extra layer is very unstable under the electron beam since the EDXS line profile, performed with a $30 \mathrm{~s}$ counting time on each point, destroyed it: each successive position of the electron beam can clearly be seen in Figure 8(a). We are convinced that this amorphous layer was present prior to the $\mathrm{BTO} / \mathrm{SRO} / \mathrm{YSZ}$ deposition and resulted from the process used for the microcantilevers fabrication on the SOI substrates. It appeared only on the bridge edge as confirmed by FIB cut imaging. The total thickness of the three silicon based layers of the microcantilever (crystalline $\mathrm{Si}$-thin silicon oxide-amorphous $\mathrm{SiOx}$ ) is $350 \mathrm{~nm}$ which is close to the expected $340 \mathrm{~nm} \mathrm{Si}(\mathrm{SOI})$ and to the measured thickness $(340 \mathrm{~nm})$ on a non-released area on the side of the same TEM lamella: this close area does not exhibit any extra amorphous layer but a single thin ( $9 \mathrm{~nm}$ thick) silicon oxide layer. The TEM observation is also consistent with the SAED pattern of the suspended stacking layers presented in Figure 7(b), which does not show well-defined dot arrays anymore. Some dots forming arcs are observed especially for the YSZ layer, which exhibit two main growth orientations: the expected [001]*, well defined, and another [1iㅣ $]^{*}$ with slight disorientations with respect to growth axis of crystalline grains. This can be expected if the oxides growth occurs on an amorphous layer. For further improvement of the epitaxial growth of oxides on the released microstructures, a particular care will have to be paid in order to get silicon terminated surfaces and not a silicon oxide terminated ones. Nevertheless, the expected textured films frameworks (YSZ, SRO, and BTO) could be identified and indexed.

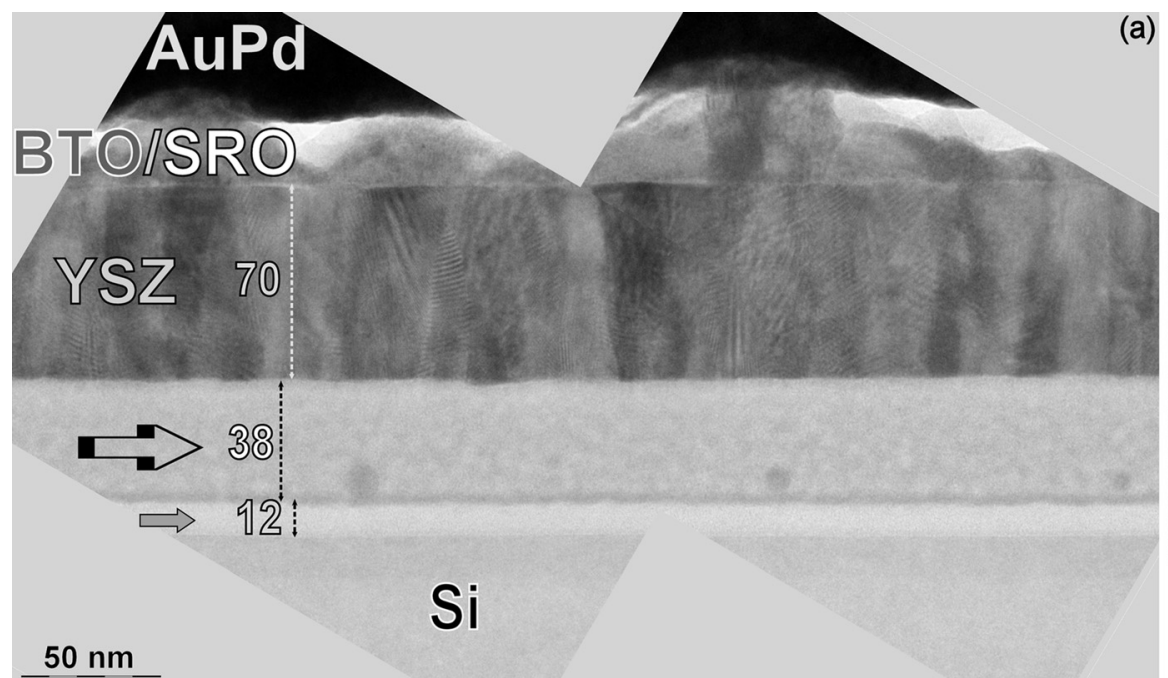

(a)

FIG. 7. (a) Medium resolution TEM image showing the polycrystalline nature of the YSZ layer, and amorphous-like contrast of an extra layer (layer thicknesses are given in $\mathrm{nm}$ ); (b) corresponding SAED pattern showing dots forming arcs for YSZ, SRO, and BTO lattices. 
(a)

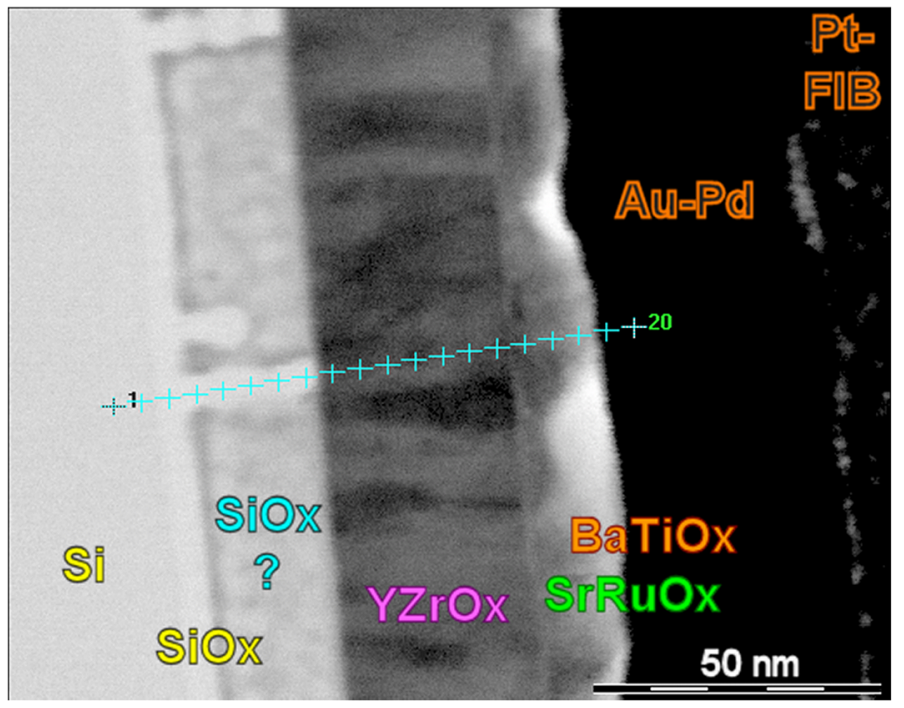

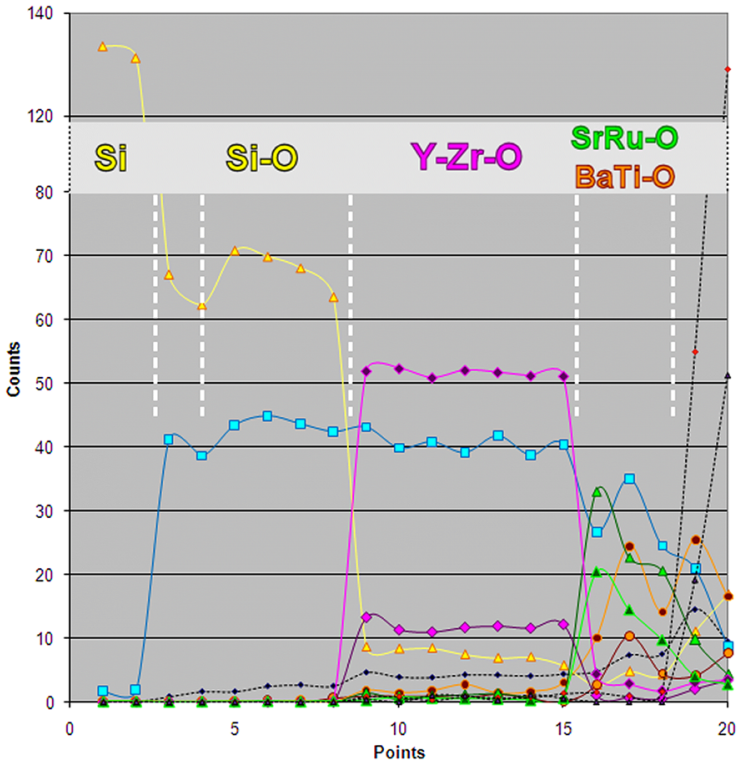

(b)

FIG. 8. (a) STEM image and (b) EDXS line profiles showing the disproportionation of the intermediate layer under the electron beam (points 4 to 8 )

\section{CONCLUSION}

(101) oriented epitaxial tetragonal $\mathrm{BaTiO}_{3}$ thin films were grown on SRO/YSZ/Si (001) by PLD at two oxygen pressures: $5.10^{-2}$ and $5.10^{-3}$ mbar, as shown by Raman spectroscopy, XRD and TEM investigations. The use of the $5.10^{-3}$ mbar $\mathrm{O}_{2}$ pressure allowed the strain relocation from inside the grains towards the grain boundaries. Thus, the $5.10^{-3}$ mbar $\mathrm{O}_{2}$ pressure was chosen to deposit the $\mathrm{BTO} / \mathrm{SRO} / \mathrm{YSZ}$ layers on silicon microcantilevers, fabricated on SOI wafers prior to the film deposition. The mechanical behaviour of the resulting BTO-based microcantilevers has been characterized by resonant frequency measurements since neither cracks nor excessive strain were observed. Young modulus of the BTO films has been estimated to be $240 \pm 18 \mathrm{GPa}$. This high value could be explained by the low film thicknesses and the small grain size as seen in the TEM study of the BTO-based released structures. The (101)-orientation of the BTO films on Si, which offers the advantage to allow either in-plane or out-plane piezoelectric actuation, depending on the targeted application, and the first characterisation of the mechanical behaviour of BTO-based microcantilevers are promising in view of MEMS and NEMS applications. The next step will be the evaluation of the BTO piezoelectric coefficient by the use of a SRO top electrode.

\section{ACKNOWLEDGMENTS}

The authors would like to acknowledge the financial support provided for this research by the Region BasseNormandie through the "Centre de compétences en nanosciences du Nord Ouest (C'nano NO)".

${ }^{1}$ P. Muralt, J. Micromech. Microeng. 10, 136 (2000).

${ }^{2}$ M. Dekkers, M. D. Nguyen, R. Steenwelle, P. M. te Riele, D. H. A. Blank, and G. Rinjders, Appl. Phys. Lett. 95, 012902 (2009).
${ }^{3}$ Y. Yoneda, T. Okabe, K. Sakaue, H. Terauchi, H. Kasatani, and K. Deguchi, J. Appl. Phys. 83, 2458 (1998).

${ }^{4}$ K. J. Choi, M. Biegalski, Y. L. Li, A. Sharan, J. Schubert, R. Uecker, P. Reiche, Y. B. Chen, X. Q. Pan, V. Gopalan, L. Q. Chen, D. G. Schlom, and C. B. Eom, Science 306, 1005 (2004).

${ }^{5}$ B. H. Hoeman, G. M. Ford, L. D. Kaufmann, and B. W. Wessels, Appl. Phys. Lett. 73, 2248 (1998).

${ }^{6}$ Y. Yano, K. Iijima, Y. Daitoh, T. Terashima, Y. Bando, Y. Watanabe, H. Kasatani, and H. Terauchi, J. Appl. Phys. 76, 7833 (1994).

${ }^{7}$ J. S. Pulskamp, A. Wickenden, R. Polcawich, B. Piekarski, M. Dubey, and G. Smith, J. Vac. Sci. Technol. B 21, 2482 (2003).

${ }^{8}$ R. B. Karabalinand, L. G. Villanueva, M. H. Matheny, J. E. Sader, and M. L. Roukes, Phys. Rev. Lett. 108, 236101 (2012).

${ }^{9}$ M. D. Nguyen, H. Nazeer, K. Karakaya, S. V. Pham, R. Steenwelle, M. Dekkers, L. Abelmann, D. H. A. Blank, and G. Rijnders, J. Micromech. Microeng. 20, 085022 (2010).

${ }^{10}$ D. Isarakorn, A. Sambri, P. Janphuang, D. Briand, S. Gariglio, J.-M. Triscone, F. Guy, J. W. Reiner, C. H. Ahn, and N. F. de Rooij, J. Micromech. Microeng. 20, 055008 (2010).

${ }^{11}$ S. H. Baek, J. Park, D. M. Kim, V. A. Aksyuk, R. R. Das, S. D. Bu, D. A. Felker, J. Lettieri, V. Vaithyanathan, S. S. N. Bharadwaja, N. BassiriGharb, Y. B. Chen, H. P. Sun, C. M. Folkman, H. W. Jang, D. J. Kreft, S. K. Streiffer, R. Ramesh, X. Q. Pan, S. Trolier-McKinstry, D. G. Schlom, M. S. Rzchowski, R. H. Blick, and C. B. Eom, Science 334, 958 (2011).

${ }^{12}$ D. Berlincourt and H. Jaffe, Phys. Rev. 111, 143 (1958).

${ }^{13}$ A. Safari and M. Abazari, IEEE Trans. Ultrason. Ferroelectr. Freq. Control. 57, 2165 (2010).

${ }^{14}$ K. Yokota, H. Morigou, and F. Miyashita, Nucl. Instrum. Methods Phys. Res. B 257, 468 (2007).

${ }^{15}$ B. Chen, H. Yang, J. Miao, L. Zhao, L. X. Cao, B. Xu, X. G. Qiu, and B. R. Zhao, J. Appl. Phys. 97, 024106 (2005).

${ }^{16}$ L. Qiao and X. Bi, J. Alloys Compd. 477, 560 (2009).

${ }^{17}$ G. Niu, B. Gautier, S. Yin, G. Saint-Girons, P. Lecoeur, V. Pillard, G. Hollinger, and B. Vilquin, Thin Solid Films 520, 4595 (2012).

${ }^{18}$ C. A. P. de Araujo, J. D. Cuchiaro, L. D. McMillan, M. C. Scott, and J. F. Scott,Nature (London) 374, 627 (1995).

${ }^{19}$ G. Asano, H. Morioka, and H. Funakubo, Appl. Phys. Lett. 83, 5506 (2003).

${ }^{20}$ W. Wu, K. H. Wong, C. L. Choy, and Y. H. Zhang, Appl. Phys. Lett. 77, 3441 (2000).

${ }^{21}$ Y. A. Boikov and T. Claeson, J. Appl. Phys. 89, 5053 (2001).

${ }^{22}$ K. Watanabe, M. Ami, and M. Tanaka, Mater. Res. Bull. 32, 83 (1997).

${ }^{23}$ G. Niu, S. Yin, G. Saint-Girons, B. Gautier, P. Lecoeur, V. Pillard, G. Hollinger, and B. Vilquin, Microelectron. Eng. 88, 1232 (2011).

${ }^{24}$ C. Dubourdieu, J. Bruley, T. M. Arruda, A. Posadas, J. Jordan-Sweet, M. M. Frank, E. Cartier, D .J. Frank, S. V. Kalinin, A. Demkov, and V. Narayanan, Nat. Nanotechnol. 8, 748 (2013). 
${ }^{25}$ M. Scigaj, N. Dix, I. Fina, R. Bachelet, B. Warot-Fonrose, J. Fontcuberta, and F. Sanchez, Appl. Phys. Lett. 102, 112905 (2013).

${ }^{26}$ S. Y. Hou, J. Kwo, R. K. Watts, J.-Y. Cheng, and D. K. Fork,Appl. Phys. Lett. 67, 1387 (1995).

${ }^{27}$ L. Méchin, J.-C. Villégier, G. Rolland, and F. Laugier, Physica C 269, 124 (1996).

${ }^{28}$ L. Méchin, P. Perna, C. Barone, J.-M. Routoure, and Ch. Simon, Mater. Sci. Eng. B 144, 73 (2007).

${ }^{29}$ P. Perna, L. Méchin, M. P. Chauvat, P. Ruterana, Ch. Simon, and U. Scotti di Uccio, J. Phys.: Condens. Matter 21, 306005 (2009).

${ }^{30}$ C. Jorel, H. Colder, A. Galdi, and L. Méchin, IOP Conf. Ser.: Mater. Sci. Eng. 41, 012012 (2012).

${ }^{31} \mathrm{See}$ http://www.horiba.com/scientific/products/ellipsometers/software/ for DeltaPsi2 software

${ }^{32}$ T. Yaguchi, T. Kamino, T. Ishitani, and R. Urao, Microsc. Microanal. 5, 365 (1999).

${ }^{33} \mathrm{H}$. Roberts and B. Otterloo, EFUG meeting, Arcachon, France (2001).

${ }^{34}$ D. W. Carr and H. G. Craighead, J. Vac. Sci. Technol. B 15, 2760 (1997).

${ }^{35}$ A. R. Forouhi and I. Bloomer, Phys. Rev. B 34, 7018 (1986).

${ }^{36}$ G. E. Jelisson and F. A. Modine, Appl. Phys. Lett. 69, 371 (1996).

${ }^{37}$ L. Méchin, A. Chabli, F. Bertin, M. Burdin, G. Rolland, C. Vannuffel, and J.-C. Villégier, J. Appl. Phys. 84, 4935 (1998).

${ }^{38} \mathrm{~F}$. Ott, see http://www-llb.cea.fr/prism/programs/simulreflec/simulreflec.html for Simulreflec, Lab. Léon Brillouin CEA/CNRS UMR12.

${ }^{39}$ J. Roldan, F. Sanchez, C. Guerrero, F. Benitez, C. Ferrater, and M. Varela, Appl. Surf. Sci. 154, 159 (2000).

${ }^{40}$ G. Koster, L. Klein, W. Siemons, G. Rijnders, J. S. Dodge, C. B. Eom, D. H. A. Blank, and M. R. Beasley, Rev. Mod. Phys. 84, 253 (2012).

${ }^{41}$ A. Galdi, P. Orgiani, L. Maritato, and L. Méchin, J. Phys. Condens. Matter. 24, 435603 (2012).

${ }^{42}$ P. W. Forsbergh, Phys. Rev. 93, 686 (1954).

${ }^{43}$ B. W. Lee and K. H. Auh, J. Mater. Res. 10, 1418 (1995).

${ }^{44}$ M. Ring and K. L. Kavanagh, J. Appl. Phys. 94, 5982 (2003).

${ }^{45}$ M. El Marssi, F. Le Marrec, I. A. Lukyanchuk, and M. G. Karkut, J. Appl. Phys. 94, 3307 (2003).

${ }^{46}$ L. Huang, Z. Chen, J. D. Wilson, S. Banerjee, R. D. Robinson, I. P. Herman, R. Laibowitz, and S. O'Brien, J. Appl. Phys. 100, 034316 (2006).

${ }^{47}$ M. H. Frey and D. A. Payne, Phys. Rev. B 54, 3158 (1996).

${ }^{48}$ L. H. Robins, D. L. Kaiser, L. D. Rotter, P. K. Schenck, G. T. Stauf, and D. Rytz, J. Appl. Phys. 76, 7487 (1994).

${ }^{49}$ P. Wickboldt, E. Anastassakis, R. Sauer, and M. Cardona, Phys. Rev. B 35, 1362 (1987).
${ }^{50}$ H. Z. Guo, Z. H. Chen, B. L. Cheng, H. B. Lu, L. F. Liu, and Y. L. Zhou,J. Eur. Ceram. Soc. 25, 2347 (2005).

${ }^{51}$ R. Naik, J. J. Nazarko, C. S. Flattery, U. D. Venkateswaran, V. M. Naik, M. S. Mohammed, G. W. Auner, J. V. Mantese, N. W. Schubring, A. L. Micheli, and A. B. Catalan,Phys. Rev. B 61, 11367 (2000).

${ }^{52}$ U. D. Venkateswaran, V. M. Naik, and R. Naik, Phys. Rev. B 58, 14256 (1998).

${ }^{53}$ H. E. Swanson, R. K. Fuyat, and G. M. Ugrinic, NBS Circular 539, 3 (1954), data for 34 inorganic substances.

${ }^{54}$ D. Y. Kim, S. G. Lee, Y. K. Park, and S. J. Park, Jpn. J. Appl. Phys., Part 1 34, 1564 (1995).

${ }^{55}$ D. Y. Kim, S. G. Lee, Y. K. Park, and S. J. Park, Mater. Lett. 40, 146 (1999).

${ }^{56}$ N. Y. Lee, T. Sekine, Y. Ito, and K. Uchino, Jpn. J. Appl. Phys., Part 1 33, 1484 (1994).

${ }^{57}$ D. Y. Kim, S. G. Lee, Y. K. Park, and S. J. Park, Jpn. J. Appl. Phys., Part 1 36, 7307 (1997).

${ }^{58}$ W. C. Young and R. G. Budynas, Roark's Formulas for Stress and Strain (McGraw-Hill, 1989).

${ }^{59}$ M. A. Hopcroft, W. D. Nix, and T. W. Kenny, J. MEMS 19(2), 229 (2010).

${ }^{60}$ J. J. Hall, Phys. Rev. 161, 756 (1967).

${ }^{61}$ S. Gadag, G. Subbarayan, and W. Barker, J. Mater. Sci. 41, 1221 (2006).

${ }^{62}$ P. McCarthy, ICDD Grant-in-Aid (1977).

${ }^{63}$ S. Yamanaka, T. Maekawa, H. Muta, T. Matsuda, S. Kobayashi, and K. Kurosaki, J. Solid State Chem. 177, 3484 (2004).

${ }^{64} \mathrm{~W}$. Eysel and A. Geyer, ICDD Grant-in-Aid (1992).

${ }^{65}$ T. Sakakibara, H. Izu, T. Kura, W. Shinohara, H. lwata, S. Kiyama, and S. Tsuda, in Proceedings of IEEE 5th International Symposium on Micro Machine and Human Science, October 2-4, 1994, Nagoya, Japan, p. 71 (1994).

${ }^{66}$ B. L. Cheng, M. Gabay, W. Duffy, and G. Fantozzi, J. Mater. Sci. 31, 4951 (1996).

${ }^{67}$ J. J. Wang, F. Y. Meng, X. G. Ma, M. X. Xu, and L. Q. Chen, J. Appl. Phys. 108, 034107 (2010).

${ }^{68}$ M. Uludogan, T. Cagin, and W. A. Goddard, "Ab initio studies on phase behavior of barium titanate," MRS Proc. 718, D10.1 (2002).

${ }^{69}$ Y. Duan, G. Tang, L. Qin, and L. Shi, J. Alloys Compd. 507, 513 (2010).

${ }^{70}$ Y.-B. Park, M. J. Dicken, Z.-H. Xu, and X. Li, J. Appl. Phys. 102, 083507 (2007).

${ }^{71}$ G. Arlt, D. Hennings, and G. de With, J. Appl. Phys. 58, 1619 (1985). 